\title{
Nutritional factors and immune functions of gut epithelium
}

\author{
Ian R. Sanderson \\ Department of Adult and Paediatric Gastroenterology, St Bartholomew's and \\ The Royal London School of Medicine and Dentistry, Turner Street, London E1 2AD, UK
}

\begin{abstract}
The intestinal epithelium acts as a barrier to the external environment contained within the lumen of the gut. It also transports solutes for nutrition and for immunological surveillance. The present review develops the hypothesis that changes in diet, through the composition of the lumen environment, alter the expression of genes in the epithelium. These genes include those that encode for proteins that signal to the mucosal immune system. Directly changing the expression of signalling molecules in the intestinal epithelium using transgenic techniques alters immune function. For example, up regulation of the chemokine macrophage inflammatory protein-2 increases neutrophil recruitment. Furthermore, lumen molecules such as short-chain fatty acids regulate chemokine expression by epithelial cells. By this means, the epithelium acts as a transducing monolayer signalling between the contents of the intestine and the mucosal immune system.
\end{abstract}

Intestinal epithelium: Chemokine expression: Immunological surveillance: Short-chain fatty acids

The intestinal epithelium acts as a barrier to the external environment contained within the gut lumen (Sanderson \& Walker, 1999). The barrier is not complete, as the intestine allows macromolecules to be sampled (Sanderson \& Walker, 1993) and actively absorbs nutrients. It has become increasingly realised that the enterocyte itself acts as an immune cell. For example, it has receptors for bacterial products (Naik et al. 2001), as well as expressing a wide variety of molecules on its surface that contain immunoglobulin domains. The epithelial cell also expresses proteins that may interact with immunocytes within the intestine. These proteins include surface molecules such as class II major histocompatibility complex and cytokines that are released from the epithelium such as chemokines or interleukin (IL) 6.

These signalling proteins enable the epithelial cell to orchestrate events in the intestine. In our research group we have hypothesised that changes in the intestinal lumen regulate the expression of signalling molecules by the epithelial cell. By this means, the dietary effects on the intestinal lumen acts through the epithelium to alter indirectly events in the intestine, particularly those of the mucosal immune system.

The present review will first present evidence that altering the expression of signalling genes in the epithelium affects the mucosal immune system. Second, it will describe how changes in the intestinal lumen (influenced by diet) alter the expression of these genes. The effects of the lumen on epithelial cell gene expression can, therefore, be considered as an afferent limb in this process; the effects of the epithelial cell on the mucosal immune system is the efferent limb.

\section{The efferent limb}

Evidence for the effect of epithelial cell gene expression on the mucosal immune system has come from the ability to selectively alter the expression of genes in the intestinal epithelial cell by transgenic techniques. We have used chemokine expression by the epithelium as a model to show that the epithelium can orchestrate the mucosal immune system. The chemokine IL-8, which in man results in recruitment of neutrophils, was the first identified chemotatic cytokine. However, IL-8 is not expressed in the mouse. To examine the effects of chemokines on the mucosal immune system, a system was developed whereby the chemokine macrophage inflammatory protein-2 (MIP-2), whose effects are very similar to those of IL-8 in man, was linked to a fatty acid-binding protein of the intestine (FABPI) promoter (Ohtsuka et al. 2001). The promoter is 

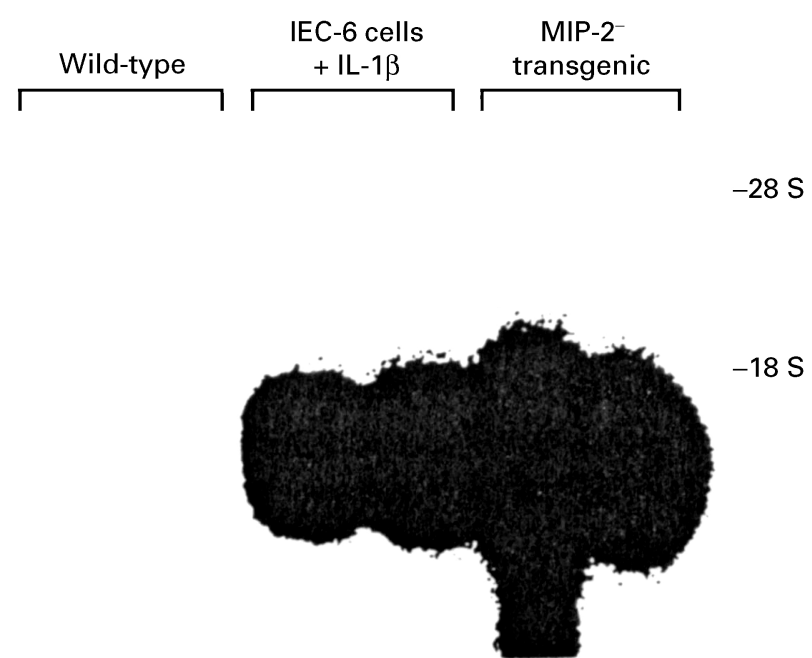

Fig. 1. Northern blot analysis of macrophage inflammatory protein2 (MIP-2) mRNA accumulation in the intestinal epithelium of wild-type and transgenic mice. MIP-2 mRNA was detected using an MIP-2 cDNA probe. Epithelial cells were removed from wild-type mice and from transgenic mice. MIP-2 mRNA derived from IEC- 6 cells stimulated with interleukin (IL) $1 \beta$ was used as a positive control. (Reproduced from Ohtsuka et al. 2001, with permission.)

only active in the epithelial cells of the small intestine and proximal colon. A construct was developed where the FABPI promoter and MIP-2 cDNA was linked to an intron and a polyadenylation site. This construct was injected into mouse oocytes. The epithelium from the first generation of the founder was shown to express MIP-2 mRNA (Fig. 1). Analysis showed effects both on neutrophil and on lymphocyte recruitment. The transgenic mice had an increased recruitment of neutrophils into the lamina propria (Fig. 2), and into the epithelial cell fraction (data not shown). The effects of the chemokine could be seen only in those tissues where the FABPI promoter was active (Fig. 2).

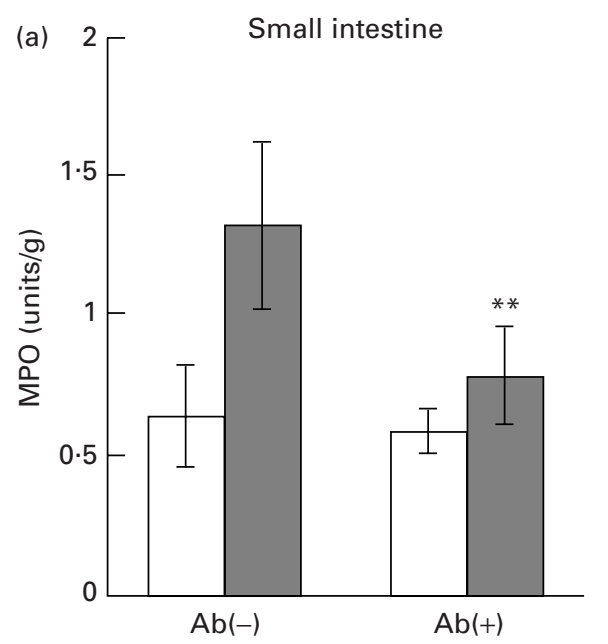

In the small intestine where the FABPI promoter is active the neutrophil recruitment, expressed as myeloperoxidase activity (per unit weight of intestine), was significantly greater $(P<0 \cdot 01)$ in the transgenic mice. In the proximal colon where the FABPI promoter is also active there was also an increase in neutrophil infiltration. However, in the distal colon where the FABPI promoter is inactive there was no effect. In addition, the liver and the spleen showed no increased infiltration in the transgenic mouse compared with the normal mouse. The FABPI promoter is not active in these organs. These data show for the first time that the epithelial cell can, through the release of chemokines, alter the mucosal immune function of the intestine in vivo.

Further analysis of the immune system demonstrated that the small intestine had increased lymphocyte infiltration, in addition to neutrophils. Lymphocyte numbers in the lamina propria were significantly increased $(P<0.05)$, and there was also a doubling of the numbers of intra-epithelial lymphocytes. The increase of intra-epithelial lymphocytes was due to an increase in $\alpha \beta$ lymphocytes and in $\gamma \delta$ lymphocytes. Further examination of the receptors on the surface of the intra-epithelial lymphocytes showed that they expressed the CXCR2, which is the receptor responsible for MIP-2 activity. These experiments show, therefore, that altering the expression of only one chemokine in the epithelium has marked effects on both lymphocyte and neutrophil function. However, changes in the intestinal lumen may affect many chemokines as well as other cytokines which alter immune function. It is likely, therefore, that the changes in gene expression in the epithelium have far-reaching effects on the rest of the mucosal immune system.

\section{The afferent limb}

The preceding section provided evidence that the epithelium can orchestrate the events of the mucosal immune system. It

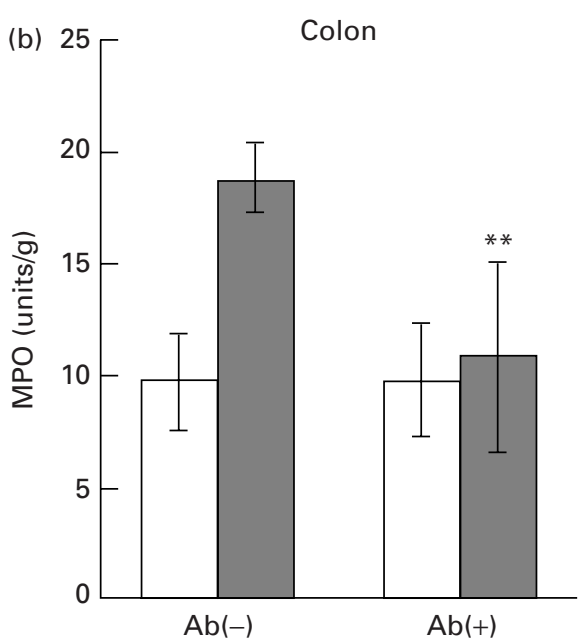

Fig. 2. Neutrophil recruitment (expressed as total myeloperoxidase (MPO) activity per unit weight of tissue) was increased on expressing macrophage inflammatory protein-2 (MIP-2) in the intestinal epithelium in both the small intestine (a) and colon (b). These effects were blocked with an MIP-2 antibody (Ab). ( $\square$ ), Wild-type mice; (@), MIP-2-transgenic mice; $A b(-)$, without $A b ; A b(+)$, with $A b$. Data are mean values and standard deviations represented by vertical bars for eight animals per group. Mean values were significantly different from those for the corresponding $A b(-)$ group: ${ }^{\star \star} P<0.01$. (Reproduced from Ohtsuka et al. 2001, with permission.) 


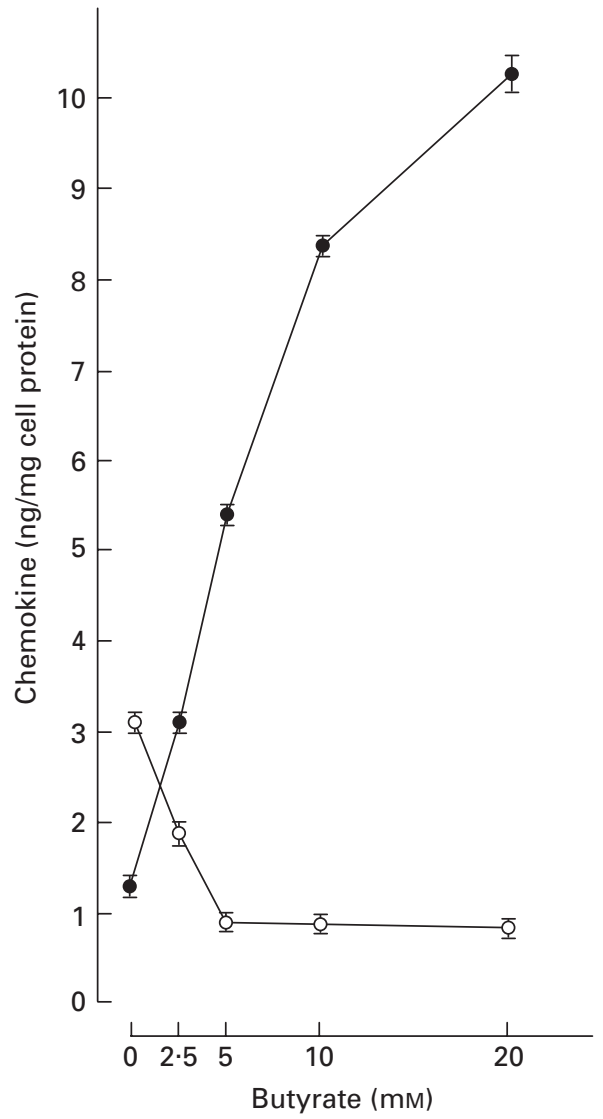

Fig. 3. Effect of interleukin (IL) $1 \beta$ and butyrate on IL-8 (•) and monocyte chemotactic protein-1 (MCP-1; O) secretion by Caco-2 cells. IL-1 $\beta$ alone stimulated the secretion of both IL-8 and MCP-1. Butyrate differentially regulated the pattern of chemokine secretion in IL-1 $\beta$-stimulated cells; IL-8 secretion was increased $(P<0.0001)$, whereas MCP-1 secretion was decreased $(P<0.0001)$. Values are means and standard deviations represented by vertical bars for three different wells. The data are representative of five experiments. (Reproduced from Fusunyan et al. 1999, with permission of Picower Institute Press.)

is the purpose of this section to show that alterations in the intestinal lumen can affect the expression of these genes. In our laboratory we have examined three sets of genes in intestinal epithelium (Sanderson \& Naik, 2000) and studied how lumen factors can alter their expression. These factors include the major histocompatibility complex class II (Sanderson et al. 1993), the insulin-like growth factorbinding protein complex and chemokine expression (Oguch et al. 1994; Nishimura et al. 1998). In the present review we will limit the evidence to the effect of lumen factors on chemokine expression (Ohno et al. 1997; Fusunyan et al. 1999).

Bacterial fermentation in the small intestine results in short-chain fatty acid production. Butyrate levels therefore reflect changes in bacterial populations and in the substrates available for bacterial metabolism. Butyrate levels vary greatly in response to external changes. For example, newborn babies have very low butyrate levels in either the small or large intestine. However, with time butyrate levels rise, reaching adult levels by 2 years of age (Midtvedt \& Midtvedt, 1992). Interestingly, butyrate levels are much (a)

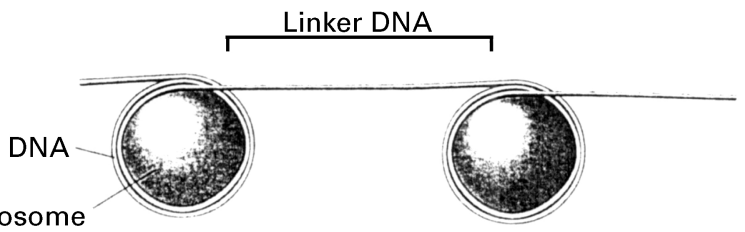

Nucleosome

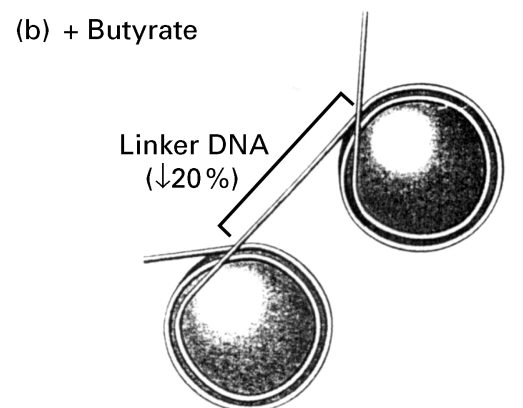

Fig. 4. Relationship between nucleosomes and DNA. (a) DNA is wrapped two full turns around nucleosomes made up of non-acetylated histones. (b) With butyrate-induced acetylation, the nucleosome expands, reducing the number of turns of DNA around the nucleosome to 1.8 , with less linker DNA connecting each nucleosome. The result of this process is that DNA cannot pass linearly from nucleosome to nucleosome, but turns at an angle after every nucleosome, leading to disruption of nucleosome packaging. $\downarrow$, Decrease. (Reproduced from Sanderson \& Naik, 2000, with permission.)

higher in bottle-fed babies than they are in breast-fed babies during the first 6 months of life (Midtvedt \& Midtvedt, 1992).

Butyrate levels therefore reflect events in the intestinal lumen, and we hypothesised that their concentrations may alter epithelial cell signalling. We therefore examined its effects on IL-8 and monocyte chemotactic protein-1 (MCP1) expression (Sanderson et al. 1987). Increasing the concentration of sodium butyrate increased IL-8 secretion while simultaneously decreasing MCP-1 expression. These effects were seen in resting epithelial cell lines, but were much more marked in cells that have been stimulated with a pro-inflammatory agent such as lipopolysaccharide or IL-1 $\beta$ (Fig. 3).

It is known that sodium butyrate alters histone acetylation. The nucleosome consists of a solenoid of histones wrapped around by an integral of two turns of DNA (Fig. 4). Butyrate increases histone acetylation, thus reducing the compactness of the histone. The DNA cannot wrap around the large nucleosome in an integral number of turns. The nucleosome can no longer be packaged into tight bundles. Thus, the DNA is exposed and made more amenable to transcription factors. We hypothesised that butyrate altered the expression of chemokines by this process. To test this hypothesis we used a fungicide, trichostatin A (TSA), which is 700 times more potent in inducing histone acetylation than butyrate. If the effects of butyrate on chemokine secretion were due to increased histone acetylation, we would expect them to be reproduced by the TSA. Experiments with TSA (Fig. 5) showed that TSA increased IL-8 secretion and decreased MCP-1 secretion. 


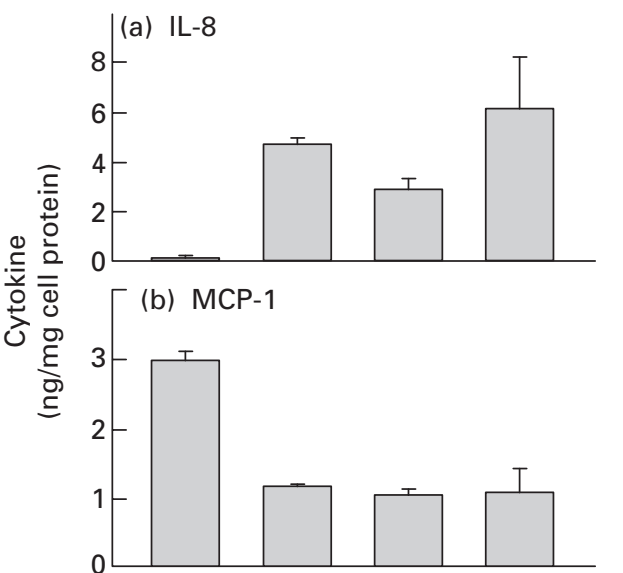

(c) Histones

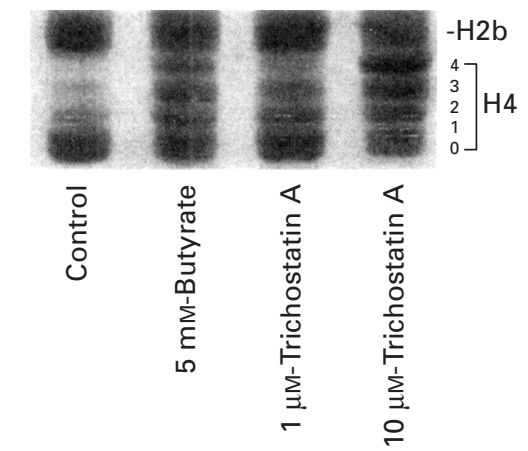

Fig. 5. Effect of butyrate and trichostatin-A in the induction of histone acetylation and chemokine secretion by Caco-2 cells. Both butyrate and trichostatin $A$ increased the acetylation of histones (c). Interleukin (IL) 8 secretion was simultaneously increased $(P<0.0001$; a) and MCP-1 production decreased in Caco-2 cells stimulated with IL-1 $\beta(P<0.001 ; b)$. Trichostatin-A, a specific histone deacetylase inhibitor, acted in a manner similar to that of butyrate when given at concentrations that produced a comparable change in histone acetylation. Values are means and represented by vertical bars for three different wells. The data are representative of three experiments. (Reproduced from Fusunyan et al. 1999, with permission.)

Fig. 5(c) shows that both TSA and butyrate increased the acetylation of histone 4 . Non-acetylated histones move rapidly through the gel and form a single band, whereas acetylated histones form a ladder depending on the extent of acetylation. Histone 4 has four lysine residues which are acetylated, and thus acetylation of histones will result in a ladder of five bands. This type of ladder can be seen in the cells given butyrate or TSA. Fig. 5(a and b) show that TSA has a similar effect to butyrate; it increases IL-8 secretion and decreases MCP-1 secretion. Furthermore, the extent of this increase in IL- 8 varies with the extent of histone acetylation. Moreover, the effect of butyrate on histone acetylation was reversible (data not shown). After removal of butyrate, histone acetylation returns to normal and the effects of IL- $1 \beta$ on IL- 8 secretion and MCP- 1 secretion return to those seen in the untreated cells. Different length short-chain fatty acids have differing effects on histone acetylation. Butyrate is the most effective short-chain fatty acid in inducing histone acetylation, while longer and shorter $\mathrm{C}$ lengths have lesser effects. This effect on histone acetylation is reflected by effects on the expression of IL-8
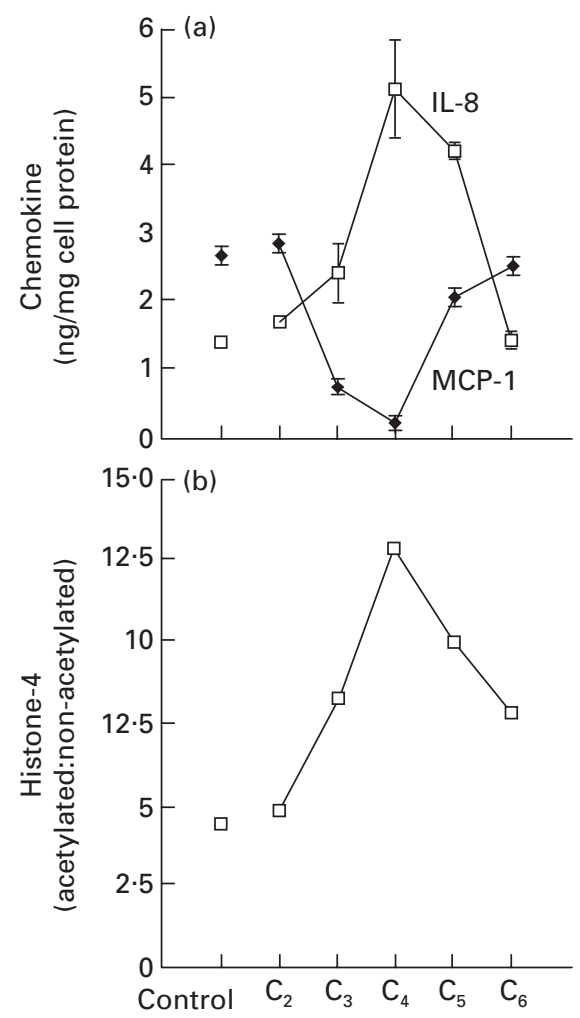

Fig. 6. Short-chain fatty acids of different chain lengths $\left(C_{2}-C_{6}\right)$ alter interleukin $8(\square)$ and monocyte chemotactic protein-1 $(\bullet)$ production (a) according to their effects on histone acetylation (b). Here $5 \mathrm{~mm}$ each of acetate $\left(C_{2}\right)$, propionate $\left(C_{3}\right)$, butyrate $\left(C_{4}\right)$, valerate $\left(C_{5}\right)$, and hexanoate $\left(\mathrm{C}_{6}\right)$ were added to Caco-2 cells for $24 \mathrm{~h}$. Interleukin $1 \beta$ was then given for a further $24 \mathrm{~h}$. Acetate and hexanoate had little effect on acetylation and on chemokine expression (NS). Valerate and propionate altered both chemokine secretion $(P<0.01)$ and histone acetylation, but the effects were less than those of butyrate. Values are means and standard deviations represented by vertical bars for three different wells. The data are representative of three experiments. (Reproduced from Fusunyan et al. 1999, with permission of Picower Institute Press.)

and MCP-1 (Fig. 6). Butyrate elicits the greatest increase in IL- 8 secretion and the greatest decrease in MCP-I secretion of the various short-chain fatty acids used.

In summary, these experiments show that sodium butyrate alters the expression of chemokines in the epithelial cell. In addition, short-chain fatty acids alter this expression through histone acetylation. These experiments, however, do not exclude the possibility that additional effects of sodium butyrate may occur through promoter systems. It is a challenge of future work to examine the interaction between chromosomal regulation, as seen in these experiments, and promoter-based regulation with both butyrate and other lumen molecules.

\section{Conclusions}

Our experiments have examined the hypothesis that changes in the intestinal lumen can alter the expression of signalling molecules in the intestine epithelium that direct the mucosal immune system. Thus, not only are there specialised organs that sense the environment signalling information via the 
nervous system to the brain, but also the lining of the intestine acts as a relay for transducing the information of the intestinal environment to the mucosal immune system. This mechanism has advantages over other forms of immune surveillance in the gut that require the breach of the mucosal barrier. Such breaches can be manipulated by invading organisms to enter the body. The classic example of this process is the polio virus, which enters the intestine through the M-cell to the immune system of Peyer's patch which is designed as a sampling system of the mucosal environment.

We believe that these signalling processes are important not only in health but also in the treatment of disease. For example, the primary therapy of children with Crohn's disease in the UK is treatment with enteral feeds (Sanderson et al. 1987a,b). Although there are many mechanisms by which enteral feeds may be effective, we think it possible that one of them is by radically altering the environment of the lumen to such an extent that it varies the signals from the intestinal epithelium to the mucosal immune element. This change results in a down regulation of the inflammation of Crohn's disease. By this means there is a decrease in the inflammatory activity of Crohn's disease.

\section{References}

Fusunyan RD, Quinn JJ, Fujimoto M, MacDermott RP \& Sanderson IR (1999) Butyrate switches the pattern of chemokine secretion by intestinal epithelial cells through histone acetylation. Molecular Medicine 5, 631-640.

Midtvedt AC \& Midtvedt T (1992) Production of short chain fatty acids by the intestinal microflora during the first 2 years of human life. Journal of Pediatric Gastroenterology and Nutrition 15, 395-403.

Naik S, Kelly EJ, Meijer L, Pettersson S \& Sanderson IR (2001) Absence of Toll-like receptor 4 explains endotoxin hypo- responsiveness in human intestinal epithelium. Journal of Pediatric Gastroenterology and Nutrition 32, 449-453.

Nishimura A, Fujimoto M, Oguchi S, Fusunyan RD, MacDermott RP \& Sanderson IR (1998) Short chain fatty acids regulate IGF binding protein secretion by intestinal epithelial cells. American Journal of Physiology 275, E55-E63.

Oguchi S, Walker WA \& Sanderson IR (1994) Profile of IGFbinding proteins secreted by human intestinal epithelial cells changes with differentiation. American Journal of Physiology 267, G843-G850.

Ohno Y, Lee J, Fusunyan RD, MacDermott RP \& Sanderson IR (1997) Macrophage inflammatory protein-2: chromosomal regulation in rat intestinal epithelial cells. Proceedings of the National Academy of Sciences USA 94, 10279-10284.

Ohtsuka Y, Lee J, Stamm DS \& Sanderson IR (2001) MIP-2 secreted by epithelial cells increases neutrophil and lymphocyte recruitment in the mouse intestine. Gut (In the Press).

Sanderson IR, Boulton P, Menzies I \& Walker-Smith JA (1987a) Improvement of abnormal lactulose/rhamnose permeability in active Crohn's disease of the small bowel by an elemental diet. Gut 28, 1073-1076.

Sanderson IR \& Naik S (2000) Dietary regulation of intestinal gene expression. Annual Review of Nutrition 20, 311-338.

Sanderson IR, Ouellette AJ, Carter EA \& Harmatz PR (1993) Ontogeny of Ia messenger RNA in the mouse intestinal epithelium is modulated by age of weaning and diet. Gastroenterology 105, 974-980.

Sanderson IR, Udeen S, Davies PSW, Savage MO \& Walker-Smith JA (1987b) Remission induced by an elemental diet in small bowel Crohn's disease. Archives of Disease in Childhood 62, 123-127.

Sanderson IR \& Walker WA (1993) Uptake and transport of macromolecules by the intestine: Possible roles in clinical disorders (an update). Gastroenterology 104, 622-639.

Sanderson IR \& Walker WA (1999) Mucosal barrier. In Handbook of Mucosal Immunology, 2nd ed., pp. 5-17 [R Ogra, J Mestecky, J McGhee, J Bienenstock, M Lamm and W Strober, editors.] London: Academic Press. 